\title{
Quantum Mechanical Studies on the Mode of Hydrogen Bonding, Stability and Molecular Conformations of Phenol-Water Complexes
}

\author{
Mrinal Jyoti Bezbaruah, Shruti Sharma, Benzir Ahmed, Bipul Bezbaruah* \\ Department of Applied Sciences, Gauhati University, Guwahati, India \\ Email: ^bipulbezbaruah@gmail.com
}

How to cite this paper: Bezbaruah, M.J., Sharma, S., Ahmed, B. and Bezbaruah, B. (2019) Quantum Mechanical Studies on the Mode of Hydrogen Bonding, Stability and Molecular Conformations of Phenol-Water Complexes. Computational Chemistry, 7, 59-71.

https://doi.org/10.4236/cc.2019.73005

Received: March 18, 2019

Accepted: July 1, 2019

Published: July 4, 2019

Copyright $\odot 2019$ by author(s) and Scientific Research Publishing Inc. This work is licensed under the Creative Commons Attribution International License (CC BY 4.0).

http://creativecommons.org/licenses/by/4.0/

(c) (i) Open Access

\begin{abstract}
It is experimentally well established that the phenolic systems such as phenol and diphenols undergo strong hydrogen bonding interaction with water molecule. But, the possible mode hydrogen bonding in phenol-water systems may be of different types. Although, the experimental methods are not always well enough to give the proper hydrogen bonding conformations in the phenol-water complexes. The hydrogen bonding ability in phenol-water systems can directly be influenced by changing the interacting sites in the given molecular systems, which could be investigated by theoretical studies. Generally, in phenol-water system, the hydrogen bonding is taking place through $-\mathrm{OH}$ group of phenol with water molecule, and this kind of interactions between phenol-water and diphenol-water complexes have been extensively investigated in electronic ground state by Quantum Mechanical MP4 calculations. It is also very important to study the stability of different phenol-water complexes and to find out the proper phenol-water complexes with minimized interaction energy. This study will also be helpful for understanding the effect of hydrogen bonding interaction in a better way on other aromatic systems.
\end{abstract}

\section{Keywords}

Phenol, Diphenol, MP4 Method, Hydrogen Bonding

\section{Introduction}

Non-covalent and hydrogen bonding interactions are very important and essential for modern chemistry, material sciences, molecular biology, etc. These interactions are quite common in aromatic-pi systems and these kinds of forces can exist as pi-pi stacking, pi-cations, pi-anions, etc. Among these non-covalent in- 
teractions, the hydrogen bonding interactions are the most important in different molecular system as they play a very crucial role in the formation of fundamental units of bulky molecules [1] [2] [3] [4]. It is very common that phenol and diphenol molecule shows hydrogen bonding with water molecule through its functional group $(-\mathrm{OH})$. But, interestingly the mode of hydrogen bonding between these systems is also a very important factor. Therefore, the formation of intermolecular hydrogen bonding with other chemical species or solvent molecules plays a crucial role in its molecular properties such as bond lengths, bond angles and electron density on atoms which further influence on the chemical reactivity of phenolic systems [5] [6]. The determination and quantification of such types of hydrogen bonding or non-covalent interaction are highly desirable in order to predict and understand the probable molecular interactions or concerned reaction mechanism of molecular association [7]. Moreover, the non-covalent interactions are often low in energy $(1-5 \mathrm{kcal} / \mathrm{mol})$ as compare to the covalent interactions $(80-110 \mathrm{kcal} / \mathrm{mol})$ [8].

Nowadays, people have been investigating to identify the hydrogen bond motifs, one of the main aims is to expand the available list of hydrogen bond donor and acceptor in the periodic table for a wider range of application [9] [10] [11] [12] [13]. A number of molecular complexes have extensively been studied by scientists exhibiting D-H---A interactions, where D-H is the "H-bond" Donor and A is the Acceptor both experimentally and computationally [14] [15] [16] [17]. Computational calculations also show that there exists a significant interaction between a hydrogen bond donor and the centre of an aromatic ring, which acts as a hydrogen bond acceptor. This interaction is as strong as a normal hydrogen bond, which contributes approximately $3 \mathrm{kcal} / \mathrm{mol}(1 \mathrm{cal}=4.184 \mathrm{~J})$ of stabilizing energy and it plays a significant role in molecular associations [18]. Studies on hydrogen bonding interaction with common aromatic systems are well known, for example, H-bonding between electron donor and aromatic ring acceptor was studied by Ray L. Brinkley et al. [19]. The interaction energy of intermolecular phenol-water complex is in the range of about $15-28 \mathrm{kcal} / \mathrm{mol}$, which is about three times higher compared to its analogue i.e. O-H---Se [20]. Even $\mathrm{O}-\mathrm{H}---\mathrm{S}$ hydrogen bond interaction energy in phenol- $\mathrm{SH}_{2}$ complex is higher than that of O-H---Se in Phenol- $\mathrm{SeH}_{2}$ complex. The stabilization energy of $\mathrm{O}-\mathrm{H}---\mathrm{O}$ hydrogen bond was estimated to be similar to its analogue i.e. $\mathrm{O}-\mathrm{H}---\mathrm{Se}$ and O-H---S [21]. It is well known for classical Hydrogen bond interaction that the introduction of a positive charge into the proton donor or negative charge on the acceptor enhanced the stabilization energy [22]. Luminol system was also well studied for their extended hydrogen bonding network in water solution [23]. In some cases, it has been observed that the hydrogen bonding interaction can also affected by infrared spectra at high pressure and temperature, e.g. $\pi$-hydrogen bonding interaction between water and aromatic hydrocarbons (like benzene, toluene, ethylbenzene, xylene, and mesitylene) [24]. In this current work, we have studied hydrogen bonding interaction between simple aromatic phenol and diphenol rings with water for different phenol-water confor- 
mations and also study the stability of such conformers by using computational method. We also try to study more detailed investigation of this mentioned phenomenon where we could investigate on the minute details like different path of interaction, orientation of the molecules by calculating single point energy, Mullikan charge and Gibbs free energy by MPn methods.

\section{Methodology}

In our investigations all the models were constructed by using Gaussian 5.0. We have studied the hydrogen bonding interactions between simple phenolic system and water molecules by computational methods. Several phenol-water complexes were constructed in such a way that they have different orientation with respect to each other and optimized these complexes in gas phase at room temperature. The molecular structures were optimized by using Møller-Plesset perturbation methodology i.e. MP2 level of theory and double zeta basis set $6-311 \mathrm{G} /++(\mathrm{d}, \mathrm{p})$ at $298 \mathrm{~K}$ and 1atmospheric pressure. After optimization it gives a constant intermolecular hydrogen bond distances and then interaction energies were calculated by changing the intermolecular hydrogen bond distances for every phenol-water complexes. We have calculated the single point energy by MP4 level of theories with basis set $6-311++\mathrm{G}(\mathrm{d}, \mathrm{p})$. For our studies we also calculated Gibbs free energy, Mullikan charges etc. for each phenol-water complexes [25].

The interaction energies $\left(\mathrm{E}_{\mathrm{IE}}\right)$ are calculated by the following equation;

$$
\mathrm{E}_{\mathrm{IE}}=\mathrm{E}_{\text {Phenol-water }}-\mathrm{E}_{\text {Phenol }}-\mathrm{E}_{\text {water }}
$$

Here, $\mathrm{E}_{\text {Phenol-water }}=$ Energy for phenol-water complex, $\mathrm{E}_{\text {Phenol }}=$ Energy of Phenol, $\mathrm{E}_{\text {water }}=$ Energy of water.

\section{Result and Discussions}

In this current study, we have prepared different phenol-water models to analyze the actual hydrogen bonding interactions, stability and effect in phenol-water complexes. Phenol can directly interact with water in many ways, but we have taken only the most prominent and chemically favoured interactions between the water and phenol molecules. As we know, phenol undergoes strong hydrogen bonding interactions with its neighbouring water molecule when it is in aqueous medium and this is already experimentally established. Phenol interacts with water molecule through the $-\mathrm{OH}$ group of phenol, depending on the electron denoting and accepting abilities $-\mathrm{OH}$ group of phenol can interact with water either through $\mathrm{H}$ atom (donor) or through $\mathrm{O}$ atom (acceptor). It can also show weak $\pi$-dipole interaction i.e., $\pi$-electrons of phenol interacts with dipoles of water and many other mode of interactions. To observe the proper phenol- $\mathrm{H}_{2} \mathrm{O}$ complexes, we have constructed four different chemically favoured phenol- $\mathrm{H}_{2} \mathrm{O}$ complexes (Figure 1(a) \& Figure 1(b)). These proposed constructed phenol- $\mathrm{H}_{2} \mathrm{O}$ complexes are universally accepted and shows better interaction between phenol and water molecule. Our investigation is based on these four proposed models and to determine the proper phenol- $\mathrm{H}_{2} \mathrm{O}$ conformation with 
<smiles>OCOc1ccccc1</smiles>

Model 1<smiles>OCOc1ccccc1</smiles>

Model 3<smiles>Oc1ccccc1</smiles>

Model 2

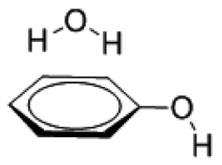

Model 4

(a)<smiles>OCOc1ccc(O)cc1</smiles><smiles>Oc1ccc(OP)cc1</smiles>

Model 1<smiles>Oc1ccc(O)cc1</smiles>

Model 3

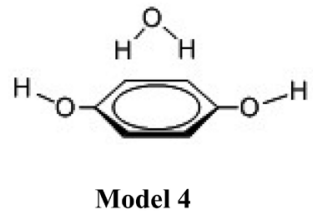

(b)

Figure 1. (a) Proposed models for phenol-water complexes; (b) Proposed models for phenol-water complexes.

appropriate phenol- $\mathrm{H}_{2} \mathrm{O}$ hydrogen bonding and dipole- $\pi$ interactions. We also carried out computational calculations to determine the stability of these phenol- $\mathrm{H}_{2} \mathrm{O}$ complexes. For all four proposed phenol- $\mathrm{H}_{2} \mathrm{O}$ complexes, the optimised geometry of model-1, 2 and 3 shows strong hydrogen bonding and gives a constant hydrogen bond distance between phenol and water molecule (Figures 2 (a)(i)-(iii)). On the other hand, only model-4 shows dipole- $\pi$ interaction, which is a very weak force of interaction (Figure 2(b)(iv)). It has been observed that the calculated hydrogen bond distances between the four proposed models of phenol-water complexes are found to be 1.8 and 2.0 Å respectively (Table 1). However, the crystal structure of phenol-water complexes reveal that the actual phenol-water hydrogen bond distance for any linear model is found to be $\sim 1.77$ $\AA$ [26], whereas for our proposed models the optimum hydrogen bond distance for model 1, 2 and 3, lies within a range from $1.8-2.0 \AA$ (Table 1). Again, we are trying to change the hydrogen bond distances of four proposed phenol- $\mathrm{H}_{2} \mathrm{O}$ optimized models within a range from 1.2 to $3.0 \AA$, to calculate the actual phenol-water interaction energies, by this way we can easily predict the strength of hydrogen bond, stability, bond dissociation energies as well as the chemical behaviour of these models. Generally, if we plot interaction energies $(\mathrm{kcal} / \mathrm{mol})$ for 


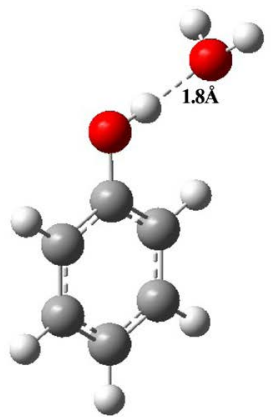

(i)

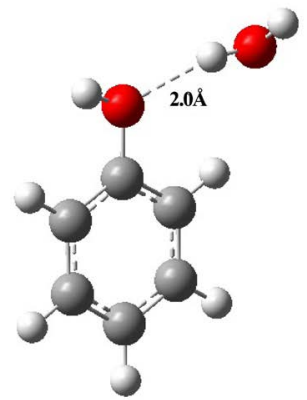

(iii)

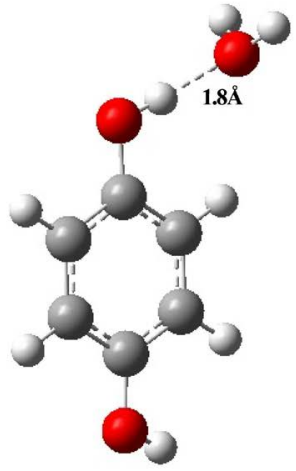

(i)

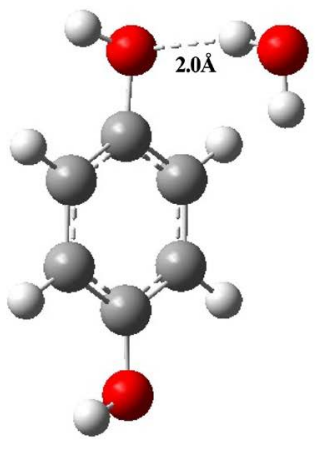

(iii)

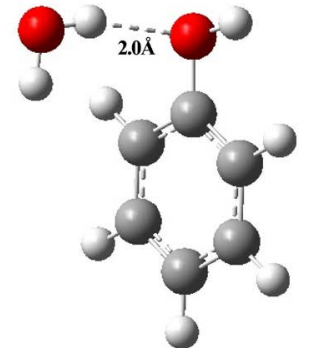

(ii)

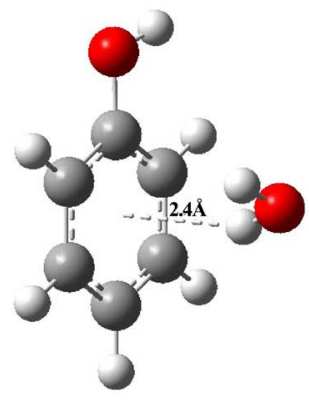

(iv)

(a)

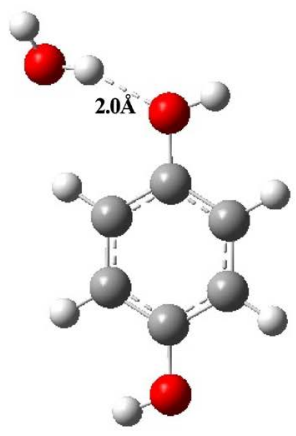

(i)

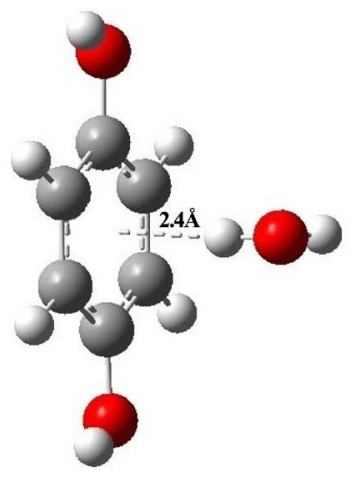

(iv)

(b)

Figure 2. (a) Minimized models with hydrogen bonding for phenol-water complexes; (b) Minimized models with hydrogen bonding for diphenol-water complexes. 
Table 1. Computed hydrogen bond distances for minimized models of phenol/diphenol-water complexes (MP4 method).

\begin{tabular}{|c|c|c|c|c|}
\hline \multirow{2}{*}{ Complexes } & \multirow{2}{*}{ Models } & \multirow{2}{*}{$\begin{array}{c}\text { Mode of Hydrogen } \\
\text { Bonding }\end{array}$} & \multicolumn{2}{|c|}{ H-Bond Distances $(\AA)$} \\
\hline & & & Theoretical & Experimenta \\
\hline \multirow{4}{*}{ Phenol-water } & 1 & $-\mathrm{OH}---\mathrm{OH}_{2}$ & 1.8 & $\sim 1.77$ \\
\hline & 2 & $\mathrm{HO}----\mathrm{H}_{2} \mathrm{O}$ & 2.0 & $\sim 1.77$ \\
\hline & 3 & $\mathrm{HO}----\mathrm{H}_{2} \mathrm{O}$ & 2.0 & $\sim 1.77$ \\
\hline & 4 & Absent & - & - \\
\hline \multirow{4}{*}{ Diphenol-water } & 1 & $-\mathrm{OH}---\mathrm{OH}_{2}$ & 1.8 & $\sim 1.77$ \\
\hline & 2 & $\mathrm{HO}----\mathrm{H}_{2} \mathrm{O}$ & 2.0 & $\sim 1.77$ \\
\hline & 3 & HO---- $\mathrm{H}_{2} \mathrm{O}$ & 2.0 & $\sim 1.77$ \\
\hline & 4 & Absent & - & - \\
\hline
\end{tabular}

each model with respect to the change in hydrogen bond distances then we can get an exponentially decreasing curve. The minimum of the curve results the more negative interaction energy value which gives the most favoured point with a stable phenol-water complex (Figure 3(a)). During the process of increasing and decreasing in hydrogen bond lengths for each model, it has been observed that on increasing the hydrogen bond length the interaction energy becomes steady and they behave as two individual molecules rather than a phenol-water complex. Moreover, on decreasing the hydrogen bond distances at a certain point the interaction between phenol-water complexes become stronger, which gives the more negative interaction energy value and it may also form covalent bond if they get closer than the minimum intermolecular distances i.e. $1.2 \AA$. Since, we have to determine the minimized interaction energy between phenol-water complexes; therefore we must take only one minimized point with more negative interaction energy value. All the minimized structures of proposed phenol-water complexes are shown in the Figures 2(a)(i)-(iv). To check the effect of solvation on the selected phenol-water complexes, we put the model in aqueous medium and we also calculated the interaction energy values of phenol- $\mathrm{H}_{2} \mathrm{O}$ complexes, and also compare it with gas phase (Figure 3(b)). Here, we can easily distinguish the change in interaction in gas phase and solvent phase i.e. in aqueous phase. The minimized interaction energies for phenol-water complexes in gas phase gives more negative value than that of the aqueous phase (Table 2). It happens because in gas phase only one type of hydrogen bonding interaction exists in the individual phenol-water complexes. But, in aqueous phase we have observed two different types of hydrogen bonding interactions viz. 1) between the individual phenol-water complexes and 2) phenol-water complexes with water molecules of the aqueous phase. In other words, it shows that the polarity of the solvent also plays an important role in the stability of the phenol-water complexes, i.e. in aqueous phase water can enhance the stability of the phenol-water complexes (Table 2). 


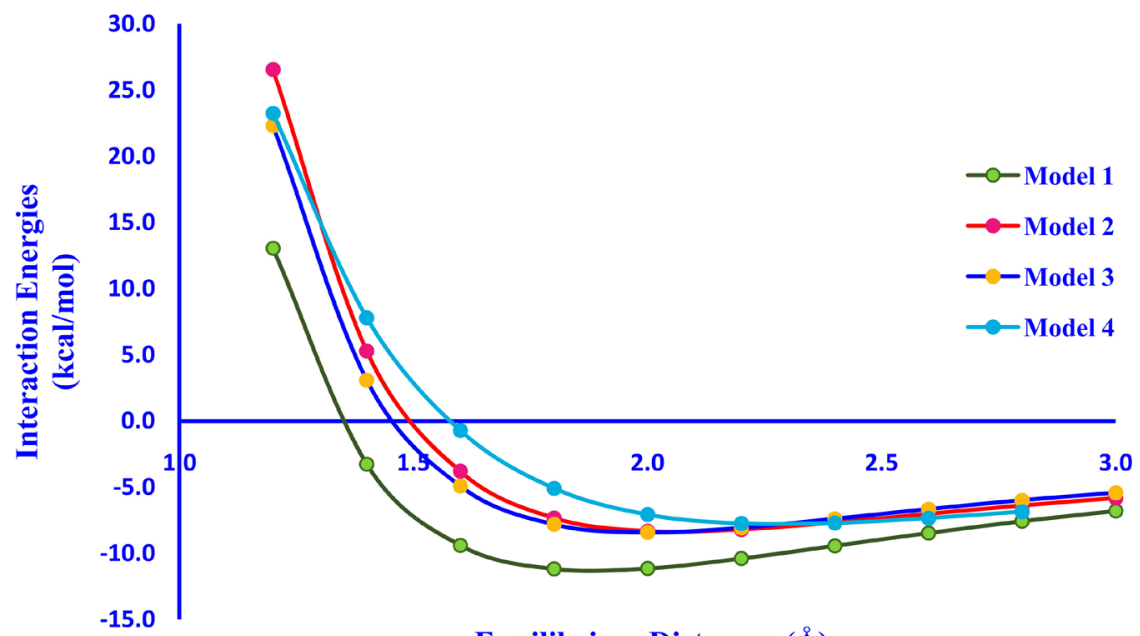

Equilibrium Distances (A)

(a)

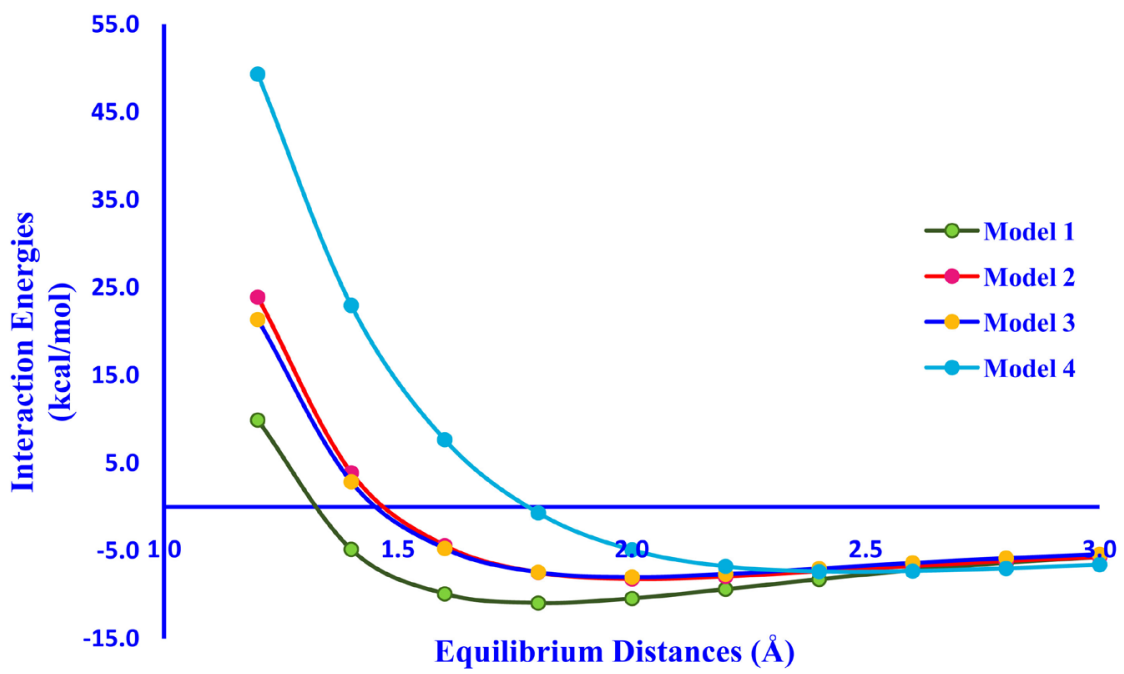

(b)

Figure 3. (a) Interaction energy plot for phenol-water complexes in gas phase; (b) Interaction energy plot for Phenol-water complexes in aqueous phase.

Table 2. Computed interaction energies for minimized models of Phenol-water complexes (MP4 method).

\begin{tabular}{cccc}
\hline \multirow{2}{*}{ Models } & \multirow{2}{*}{\begin{tabular}{c} 
Equilibrium Distances \\
\cline { 3 - 4 }
\end{tabular}} & $(\AA)$ & \multicolumn{2}{c}{ Interaction Energies $(\mathrm{kcal} / \mathrm{mol})$} \\
\cline { 3 - 4 } 1 & 1.8 & -11.1783 & Aqueous Phase \\
2 & 2.0 & -8.3149 & -10.9398 \\
3 & 2.0 & -8.4201 & -8.1831 \\
4 & 2.4 & -7.7345 & -7.9944 \\
\hline
\end{tabular}

Model-1, where the hydrogen bond occurs through - $\mathrm{OH}$ group of phenol with water molecule $\left(-\mathrm{OH}-----\mathrm{OH}_{2}\right)$, gives the most negative interaction energy value (more negative values) for both gas and solvent phase. It gives the most 
stable and stronger hydrogen bond, where the hydrogen bonds distance is found as $1.8 \AA$. The calculated $\Delta G^{\circ}$ for the minimized geometry for all four models reveals that the model-1 gives the more negative value, i.e. $-138.6503 \mathrm{kcal} / \mathrm{mol}$ (Table 3). Therefore, model-1 is the most stable and favoured conformation among the other phenol- $\mathrm{H}_{2} \mathrm{O}$ complexes. On the other hand, the study of phenol-water complexes for model- 2 and 3 are also very important, as they show similar kind of hydrogen bonding interaction $\left(\mathrm{HO}-----\mathrm{H}_{2} \mathrm{O}\right)$, with hydrogen bond distance $\sim 2.0 \AA$, but they have different conformations with respect to the water molecule as shown in Figure 2(a)(ii) \& Figure 2(a)(iii). Phenol-water complexes for model- 2 and 3 gives almost similar hydrogen bonding interaction energies for the minimized models in gas phase, which are found as -8.3149 and $-8.4207 \mathrm{kcal} / \mathrm{mol}$ respectively (Table 2). Irrespective of the above studies, in phenol- $\mathrm{H}_{2} \mathrm{O}$ model- 4 , there exists very weak dipole- $\pi$ interaction at a separation of $2.4 \AA$, as the water molecule is placed just above the centre of $\pi$-electrons of phenol ring as shown in Figure 2(a)(iv), the minimized interaction energy is found as $-7.7345 \mathrm{kcal} / \mathrm{mol}$. Although, it may also form hydrogen bond but that will be very weak as the $-\mathrm{OH}$ group of phenol and $\mathrm{H}_{2} \mathrm{O}$ placed far apart from each other. For all the phenol-water complexes, the hydrogen bonding interaction also shows similar trend in both gas and solvent phase. Again, the chemical behaviour of all the phenol-water complexes show negative $\Delta \mathrm{H}^{\circ}$ values, therefore all the systems are exothermic in nature.

Mulliken charge density calculation is also a very important tool to investigate the change in individual charge densities of the molecule before and after interaction. Greater is the change in charge densities (Mulliken charges) of the interacting individual atoms which are responsible for hydrogen bonding in phenol-water complexes, more will be the interaction in between phenol and water in that complex. The actual change in the electron charge densities of the minimized phenol-water complexes were obtained by calculating the Mulliken charges of the complex which shows whether there exist strong or weak interactions between phenol and water molecule (Table 4). In our calculations, we have obtained that the model-1 shows great change in the mulliken charge density value for $-\mathrm{OH}$ group of phenol and $\mathrm{H}_{2} \mathrm{O}$ interacting sites than that of the individual molecules. On the other hand model-2 and 3 shows weak hydrogen bonding interaction as there observed a small change in the mulliken charge

Table 3. Computed free energy change $\left(\Delta \mathrm{G}^{\circ}\right)$ and enthalpy change $\left(\Delta \mathrm{H}^{\circ}\right)$ for minimized models of phenol-water complexes (MP2 method).

\begin{tabular}{ccccc}
\hline \multirow{2}{*}{ Models } & \multicolumn{2}{c}{ Free Energy Change $\left(\Delta \mathrm{G}^{\circ}\right)(\mathrm{kcal} / \mathrm{mol})$} & \multicolumn{2}{c}{ Enthalpy Change $\left(\Delta \mathrm{H}^{\circ}\right)(\mathrm{kcal} / \mathrm{mol})$} \\
\cline { 2 - 5 } & Gas Phase & Aqueous Phase & Gas Phase & Aqueous Phase \\
\hline 1 & -138.6503 & -136.9747 & -146.3193 & -75.6230 \\
2 & -136.5839 & -133.3576 & -143.5735 & -74.6257 \\
3 & -135.9528 & -134.7559 & -143.6274 & -74.1871 \\
4 & -137.7074 & -135.9152 & -143.8150 & -74.6155 \\
\hline
\end{tabular}


Table 4. Mulliken charge calculation for phenol-water complexes (MP4 method).

\begin{tabular}{ccccccc}
\hline \multirow{2}{*}{ Models } & Medium & \multicolumn{2}{c}{ Phenol } & \multicolumn{3}{c}{ Water } \\
\cline { 3 - 7 } Phenol & Gas & -0.3713 & 0.2832 & - & - & H \\
& Aqueous & -0.4200 & 0.3169 & - & - & - \\
\multirow{2}{*}{ Water } & Gas & - & - & -0.5076 & 0.2538 & 0.2538 \\
& Aqueous & - & - & -0.5794 & 0.2897 & 0.2897 \\
\multirow{2}{*}{1} & Gas & -0.4754 & 0.4943 & -0.6026 & 0.2948 & 0.2936 \\
& Aqueous & -0.5143 & 0.5014 & -0.6335 & 0.3195 & 0.3183 \\
2 & Gas & -0.3072 & 0.2934 & -0.5474 & 0.3302 & 0.2485 \\
& Aqueous & -0.3356 & 0.3274 & -0.6124 & 0.3426 & 0.2816 \\
\multirow{2}{*}{3} & Gas & -0.3318 & 0.2844 & -0.5396 & 0.2572 & 0.2884 \\
& Aqueous & -0.3648 & 0.3199 & -0.6070 & 0.2962 & 0.3176 \\
\multirow{2}{*}{4} & Gas & -0.2576 & 0.2679 & -0.4743 & 0.2513 & 0.2642 \\
& Aqueous & -0.3070 & 0.3016 & -0.5366 & 0.2858 & 0.2726 \\
\hline
\end{tabular}

density values for the same interacting sites i.e. $-\mathrm{OH}$ group of phenol and $\mathrm{H}_{2} \mathrm{O}$ molecule (Figure 2(a)(ii) \& Figure 2(a)(iii)). In case of model-4, which shows a minute change in the Mulliken charge densities as it does not show any hydrogen bonding interaction but the existing interaction shows due to the dipole- $\pi$ interaction (Table 4).

We also carried out similar types of investigation for diphenol- $\mathrm{H}_{2} \mathrm{O}$ complexes, prepared the four models for diphenol-water complexes as constructed for phenol- $\mathrm{H}_{2} \mathrm{O}$, and also calculated the interaction energy values (Figure 1 (b) and Table 5). Interestingly, the change the interaction energies and Mulliken change densities for phenol- $\mathrm{H}_{2} \mathrm{O}$ system and diphenol- $\mathrm{H}_{2} \mathrm{O}$ systems give almost similar trend (Table 5 \& Table 6). The minimized models for diphenol-water complexes are shown in Figure 2(b). The minima of the interaction energy plots for all four diphenol-water systems, both in gas and aqueous phase shows almost similar types of interactions as it has shown in phenol-water systems (Figure 4(a) \& Figure $4(\mathrm{~b})$ ). But, these kinds of interactions might be changed on changing the substituent on phenolic systems.

\section{Conclusion}

From the above calculations, we can conclude that in model-1 hydrogen bonding is taking place through $-\mathrm{OH}-----\mathrm{OH}_{2}$, and it shows the strongest interactions in phenol-water system with hydrogen bond distance $1.8 \AA$, in both gas and water phase. It also shows the most negative interaction energy values i.e. -11.1783 $\mathrm{kcal} / \mathrm{mol}$, which reveals the most stable and favoured conformation. Model 2 and 3 also show almost similar types of hydrogen bonding but the hydrogen bond is formed through $\mathrm{HO}-----\mathrm{H}_{2} \mathrm{O}$, with a hydrogen bond distance 2.0 $\mathrm{A}$. On 


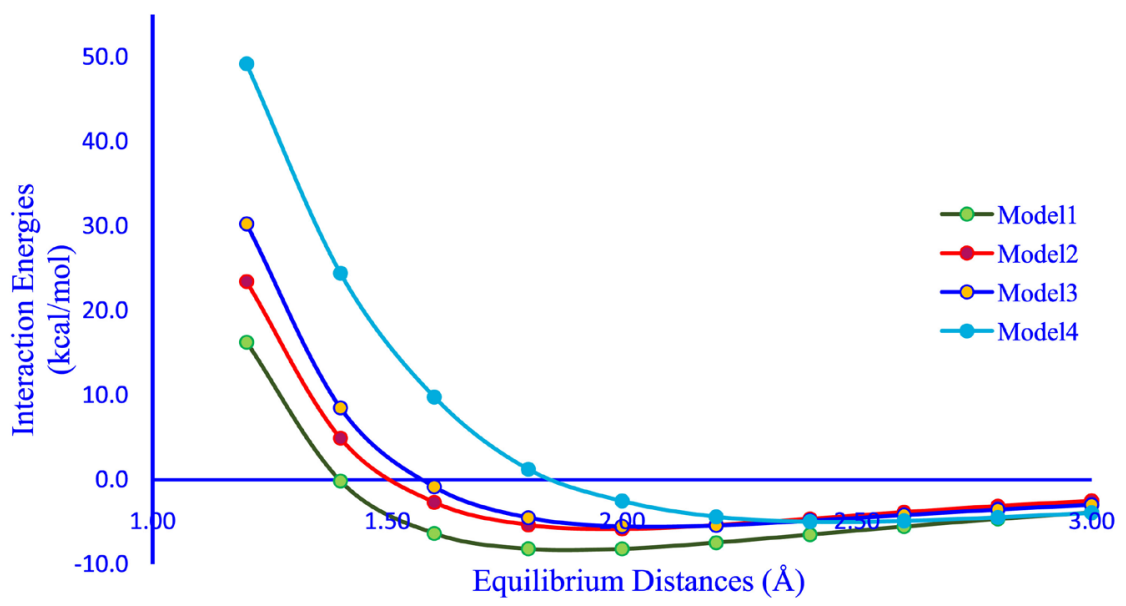

(a)

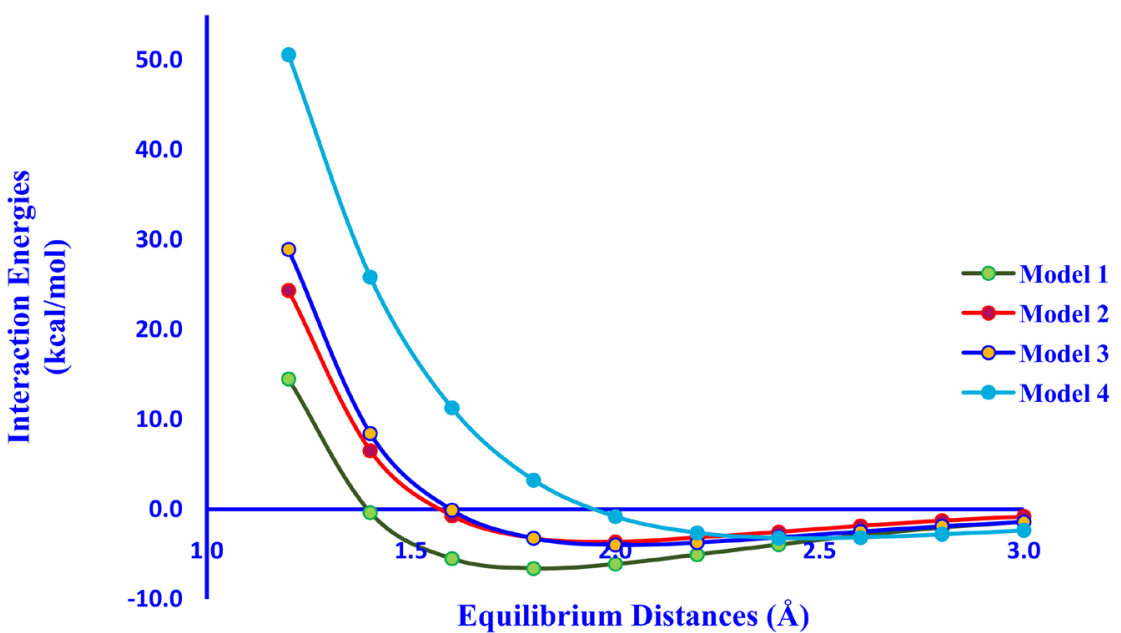

(b)

Figure 4. (a) Interaction energy plot for diphenol-water complexes in gas phase; (b) Interaction energy plot for diphenol-water complexes in aqueous phase.

Table 5. Interaction energies for minimized models of diphenol-water complexes (MP4 method).

\begin{tabular}{cccc}
\hline \multirow{2}{*}{ Models } & \multirow{2}{*}{$\begin{array}{c}\text { Equilibrium Distances } \\
(\AA)\end{array}$} & \multicolumn{2}{c}{ Interaction Energies $(\mathrm{kcal} / \mathrm{mol})$} \\
\cline { 3 - 4 } & 1.8 & Gas Phase & Aqueous Phase \\
\hline 1 & 2.0 & -8.1873 & -6.5889 \\
2 & 2.0 & -5.8227 & -3.6419 \\
3 & 2.4 & -5.5271 & -3.9699 \\
4 & & -4.9716 & -3.2049 \\
\hline
\end{tabular}

the other hand, model- 4 favourably shows dipole- $\pi$ interaction as there exists direct interaction between $\pi$-electron of phenol and dipole of water molecule at a separation of $2.4 \AA$, it gives the least negative interaction energy than the other phenol-water models. Although, we have done calculations for both gas and solvent phase systems, it has been observed that the hydrogen bond distances remain 
Table 6. Mulliken charge calculation for diphenol-water complexes (MP4 method).

\begin{tabular}{|c|c|c|c|c|c|c|c|c|}
\hline \multirow{2}{*}{ Models } & \multirow{2}{*}{ Medium } & \multicolumn{4}{|c|}{ Diphenol } & \multicolumn{3}{|c|}{ Water } \\
\hline & & O & $\mathrm{H}$ & o & $\mathrm{H}$ & O & $\mathrm{H}$ & $\mathrm{H}$ \\
\hline \multirow{2}{*}{ Diphenol } & Gas & -0.3054 & 0.2671 & -0.3054 & 0.2671 & - & - & - \\
\hline & Aqueous & -0.3559 & 0.3044 & -0.3559 & 0.3044 & - & - & - \\
\hline \multirow{2}{*}{ Water } & Gas & - & - & - & - & -0.5076 & 0.2538 & 0.2538 \\
\hline & Aqueous & - & - & - & - & -0.5794 & 0.2897 & 0.2897 \\
\hline \multirow{2}{*}{1} & Gas & -0.4866 & 0.4945 & -0.3114 & 0.2667 & -0.6024 & 0.2963 & 0.2931 \\
\hline & Aqueous & -0.5272 & 0.5020 & -0.3608 & 0.3056 & -0.6330 & 0.3209 & 0.3177 \\
\hline \multirow{2}{*}{2} & Gas & -0.3668 & 0.2823 & -0.3078 & 0.2714 & -0.5414 & 0.3029 & 0.2585 \\
\hline & Aqueous & -0.3912 & 0.3168 & -0.3583 & 0.3081 & -0.6010 & 0.3234 & 0.2940 \\
\hline \multirow{2}{*}{3} & Gas & -0.3094 & 0.2913 & -0.2959 & 0.2679 & -0.5289 & 0.2507 & 0.3161 \\
\hline & Aqueous & -0.3427 & 0.3249 & -0.3479 & 0.3043 & -0.5917 & 0.2834 & 0.3298 \\
\hline \multirow{2}{*}{4} & Gas & -0.2819 & 0.2669 & -0.2806 & 0.2672 & -0.4818 & 0.2453 & 0.2821 \\
\hline & Aqueous & -0.3331 & 0.3019 & -0.3318 & 0.3021 & -0.5436 & 0.2804 & 0.2894 \\
\hline
\end{tabular}

constant for both the phases, but the solvent phase models show more stability and negative interaction energy values, because of the polarity of the water molecule. It is also found that the diphenol-water systems show the same trends as it has shown by phenol-water system.

\section{Acknowledgements}

Authors are highly grateful to the AICTE-TEQIP-3 fund and Ministry of Human Resource Development (MHRD), New Delhi, for providing research assistance.

\section{Conflicts of Interest}

The authors declare no conflicts of interest regarding the publication of this paper.

\section{References}

[1] Salonen, L.M., Ellermann, M. and Diederich, F. (2011) Aromatic Rings in Chemical and Biological Recognition: Energetics and Structures. Angewandte Chemie International Edition, 50, 4808-4842. https://doi.org/10.1002/anie.201007560

[2] Schottel, B.L., Chifotides, H.T. and Dunbar, K.R. (2008) Anion-pi Interactions. Chemical Society Review, 37, 68-83. https://doi.org/10.1039/B614208G

[3] Ma, J.C. and Dougherty, D.A. (1997) The Cation-pi Interaction. Chemical Review, 97, 1303-1324. https://doi.org/10.1021/cr9603744

[4] Hunter, C.A., Lawson, K.R., Perkin, J. and Urch, C.J. (2001) Aromatic Interactions. Journal of the American Chemical Society, Perkin Transactions, 2, 651-669. https://doi.org/10.1039/b008495f

[5] Grabowski, S.J. (2011) What Is the Covalency of Hydrogen Bonding. Chemical Review, 111, 2597-2625. https://doi.org/10.1021/cr800346f 
[6] Kone, M., Illien, B., Laurence, C. and Graton, J. (2011) Can Quantum-Mechanical Calculations Yield Reasonable Estimates of Hydrogen Bonding Acceptor Strength? The Case of Hydrogen-Bonded Complexes of Methanol. Journal of Physical Chemistry $A, 115,13975-13985$. https://doi.org/10.1021/jp209200w

[7] Albrecht, L. and Boyd, R.J. (2012) Visualizing Internal Stabilization in Weakly Bound Systems Using Atomic Energies: Hydrogen Bonding in Small Water Clusters. Journal of Physical Chemistry A, 116, 3946-3951. https://doi.org/10.1021/jp301006g

[8] Lodish, H., Berk, A., Zipursky, S.L., Matsudaira, P., Baltimore, D. and Darnel, J. (2000) Molecular Cell Biology. 4th Edition, W. H. Freeman, New York.

[9] Biswal, H.S., Chakraborty, S. and Wategaonkar, S. (2008) Experimental Evidence of O-H-S Hydrogen Bonding in Supersonic Jet. Journal of Chemical Physics, 129, Article ID: 184311. https://doi.org/10.1063/1.3012569

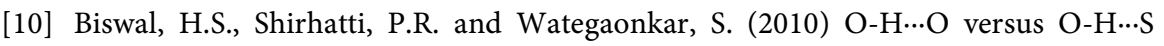
Hydrogen Bonding. 2. Alcohols and Thiols as Hydrogen Bond Acceptors. Journal of Physical Chemistry A, 114, 6944-6955. https://doi.org/10.1021/jp102346n

[11] Xu, L.H., Liu, Q., Suenram, R.D., Lovas, F.J., Walker, A.R.H., Jensen, J.O. and Samuels, A.C. (2004) Rotational Spectra, Conformational Structures, and Dipole Moments of Thiodiglycol by Jet-Cooled FTMW and Ab Initio Calculations. Journal of Moleculer Spectroscopy, 228, 243-250. https://doi.org/10.1016/j.jms.2004.04.004

[12] Pal, D. and Chakrabarti, P. (1998) Different Types of Interactions Involving Cysteine Sulfhydryl Group in Proteins. Journal of Biomolecular Structure and Dynamics, 15, 1059-1072. https://doi.org/10.1080/07391102.1998.10509001

[13] Zauhar, R.J., Colbert, C.L., Morgan, R.S. and Welsh, W.J. (2000) Evidence for a Strong Sulfur-Aromatic Interaction Derived from Crystallographic Data. Biopolymers, 53, 233-248. https://doi.org/10.1002/(SICI)1097-0282(200003)53:3<233::AID-BIP3>3.0.CO;2-4

[14] Krepps, M.K., Parkin, S. and Atwood, D.A. (2001) Hydrogen Bonding with Sulfur. Crystal Growth Design, 1, 291-297. https://doi.org/10.1021/cg015505v

[15] Francuski, B.M., Novakovic, S.B. and Bogdanovic, G.A. (2011) Electronic Features and Hydrogen Bonding Capacity of the Sulfur Acceptor in Thioureido-Based Compounds. Experimental Charge Density Study of 4-methyl-3-thiosemicarbazide. CrystEngComm, 13, 3580-3591. https://doi.org/10.1039/c0ce00760a

[16] Howard, D.L. and Kjaergaard, H.G. (2008) Hydrogen Bonding to Divalent Sulphur. Physical Chemistry Chemical Physics, 10, 4113-4118. https://doi.org/10.1039/b806165c

[17] Novakovic, S.B., Fraisse, B., Bogdanovic, G.A. and Spasojevic-De Bire, A. (2007) Experimental Charge Density Evidence for the Existence of High Polarizability of the Electron Density of the Free Electron Pairs on the Sulfur Atom of the Thioureido Group, NH-C(S)-NH2, Induced by N-H...S and C-H...S Interactions. Crystal Growth Design, 7, 191-195. https://doi.org/10.1021/cg060497+

[18] Levitt, M. and Perutz, M.F. (1988) Aromatic Rings Act as Hydrogen Bond Acceptors. Journal of Molecular Biology, 201, 751-754. https://doi.org/10.1016/0022-2836(88)90471-8

[19] Brinkley, R.L. and Gupta, R.B (2004) Hydrogen Bonding with Aromatic Ring. American Institute of Chemical Engineers, 47, 948-953.

https://doi.org/10.1002/aic.690470417

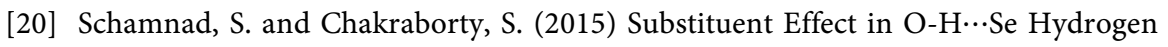
Bond-Density Functional Theory Study of Para-Substituted Phenol-SeH $\mathrm{H}_{2}$ Com- 
plexes. Chemical Physics Letters, 622, 28-33.

https://doi.org/10.1016/j.cplett.2015.01.012

[21] Harada, T., Yoshida, H., Ohno, K., Matsuura, H., Zhang, J., Iwaoka, M. and Tomod, S. (2001) Implications of Intramolecular $\mathrm{OH}$...Se Hydrogen Bonding and $\mathrm{CH}$... $\mathrm{O}$ Interaction in the Conformational Stabilization of 2-(Methylseleno)ethanol Studied by Vibrational Spectroscopy and Density Functional Theory. Journal of Physical Chemistry A, 105, 4517-4523. https://doi.org/10.1021/jp003990j

[22] Nepal, B. and Scheiner, S. (2014) Effect of Ionic Charge on the $\mathrm{CH} \cdots \pi$ Hydrogen Bond. Journal of Physical Chemistry A, 118, 9575-9587. https://doi.org/10.1021/jp5070598

[23] Xue, B., et al. (2014) Luminol: Extended Hydrogen Bond Network in Water Solution. Computational and Theoretical Chemistry, 1028, 81-86. https://doi.org/10.1016/j.comptc.2013.11.022

[24] Furutaka, S. and Ikawa, S. (1998) Hydrogen Bonding of Water with Aromatic Hydrocarbons at High Temperature and Pressure. The Journal of Chemical Physics, 108, 5159-5160. https://doi.org/10.1063/1.475953

[25] Frisch, M.J., Trucks, G.W., Schlegel, H.B., Gill, P.M.W., Johnson, B.G., Robb, M.A., Cheeseman, J.R., Keith, T., Petersson, G.A., Montgomery, J.A., Raghavachari, K., Al-Laham, M.A., Zakrzewaki, V.G., Ortiz, J.V., Foresmann, J.B., Ciolowski, J., Stefanov, B.B., Namayakkara, A., Challacombe, M., Peng, C.Y., Ayala, P.Y., Chen, W., Wong, M.W., Andres, J.L., Replogle, E.S., Gomperts, R., Martin, R.L., Fox, D.J., Binkley, J.S., Defrees, D.J., Baker, J., Stewart, J.P., Head-Gordon, M., Gonzalez, C. and Pople, J.A. (2009) Gaussian 09. Gaussian Inc., Pittsburgh.

[26] Josefin, E.U., Mikael, K., Dick, S. and Björn, Å. (2006) Water as a Hydrogen Bonding Bridge between a Phenol and Imidazole. A Simple Model for Water Binding in Enzymes. Biochimica et Biophysica Acta, 1757, 1592-1596.

https://doi.org/10.1016/j.bbabio.2006.08.005 